\title{
Coordination Analysis of Environmental and Social Problems in Transportation System
}

\author{
Zhiwen Chen \\ School of Geography and Environmental Sciences, Zhejiang Normal University, Jinhua 321004, Zhejiang Province, China \\ Correspondence should be addressed to Zhiwen Chen; zjjhczw@zjnu.edu.cn
}

Received 6 November 2020; Revised 9 December 2020; Accepted 14 December 2020; Published 23 December 2020

Academic Editor: Wei Wang

Copyright (c) 2020 Zhiwen Chen. This is an open access article distributed under the Creative Commons Attribution License, which permits unrestricted use, distribution, and reproduction in any medium, provided the original work is properly cited.

\begin{abstract}
This paper analyzes the characteristics of the transportation system and constructs a multidimensional urban public transportation evaluation index system from the perspective of basic network evaluation. The DEA efficiency evaluation model is constructed based on the perspective of environmental and social issues in transportation systems. Environmental and social issues in transportation systems refer to the differences in the average number of people carried and their technical indicators such as environmental pollution and energy consumption for different modes of transportation, which will also lead to different consumption of natural resources per unit of transportation capacity. The multidimensional public transportation system efficiency evaluation system for different evaluation scenarios provides useful technical ideas and implementation methods. To provide a basis for decision-making for transportation system planning, the operation management can help to further promote the research results in practice.
\end{abstract}

\section{Introduction}

In recent years, with the acceleration of urbanization and economic development, rapid population growth and high concentration have posed serious challenges to urban development in terms of space, energy, environment, and transportation. At the same time, rapid urbanization and motorization have given cities traffic which brings great pressure [1]. At the end of 2019, the number of motor vehicles reached 327 million, of which 240 million were cars. Compared with 2018, the annual increase was 20.85 million with an increase of $10.51 \%$. The number of motor vehicles still maintains rapid growth, with an average annual increase of 19.52 million vehicles in the past five years [2]. The excessive use of road resources by cars has brought various problems to the traffic and environment of these cities. The emission of a large amount of automobile exhaust makes the road environment continue to deteriorate $[3,4]$. The frequency of traffic safety accidents increases. Land use in various cities is restricted by the travel of motor vehicles, and residents often encounter difficulties in driving and parking
[5]. All along, local governments and public transport companies have invested a lot of money in public transport construction to improve public transport services, infrastructure, and corresponding equipment but lack an overall consideration in facility planning, financial capacity, system design, and service operations [6]. For decision-makers, the big problem in the process of managing and operating public transportation services is that the information obtained is numerous and scattered, and the information obtained lacks practical guidance on how to improve the service [7]. To enable these bus service operators and government decisionmakers to improve the quality of services, reduce operating costs, and use limited resources to obtain the maximum value needed, an effective method is needed to evaluate public transport services and can point out service improvement direction [8].

Operation speed, line delays, and comfort are key factors that affect the service level of the San Francisco BRT system. Service reliability, accessibility, travel time, linear coefficient, headway distance, and passenger flow density are six indicators that can reflect the level of public transportation 
services. The total travel time can be divided into walking to platform time, waiting time, riding time, transfer time, and potential waiting time [9]. The concept of travel cost is used to study the evaluation method of the bus system from the time allocation. The five performance criteria of bus routes, departure schedule, economy and productivity, comfort and safety, and service accessibility build a comprehensive evaluation index system [10]. The public transport performance measurement standard and evaluation system determined by the project shall be following the goals to be achieved by the public transport construction project [11]. Rous and others began to pay more attention to the concept of bus service level, highlighting indicators such as the scope of public transport services, the frequency of departures, service duration, comfort, and accessibility of pedestrian connection lines at bus stations. Multiple passenger experience indicators such as waiting time, departure frequency, bus station accessibility, punctuality, and seat comfort are used as influencing factors for judging service level [12].The four methods of excess waiting time, standard deviation of the difference between actual and scheduled headway, waiting assessment relative to departure frequency, and service punctuality relative to departure frequency all show different sensitivities to service regularity, with the excess waiting time method being the best assessment indicator. [13]. Factor analysis is used to define the variables necessary to evaluate the performance of bus transport, including the overall cost efficiency, labor utilization, vehicle utilization, service utilization, quality of service, and accessibility. Poles directly use the cost-to-benefit ratio to derive a single effectiveness measure to measure the efficiency of bus operations, but the main problem of this method is that it is difficult to measure all input and all benefits in bus operations with money [14].

Based on the above research background, establishing a set of a targeted public transport evaluation system that can reasonably evaluate the city's existing public transport system or public transport planning scheme of multiple dimensions according to different construction goals is a subject worthy of in-depth study [15]. For different planning objectives or business needs, choose a different rule layer design appropriate evaluation, taking into account the city's comprehensive public fitness between the total transport system and urban structure and development [16]. Urban virtual traffic simulation platform will be theoretical results visualization. The establishment of a comprehensive public transportation comprehensive evaluation system on the virtual platform can meet the assessment from different perspectives such as traveler, enterprise operation level, and government levels, such as achieving maximum public satisfaction or reducing noise and air pollution [17]. The comprehensive evaluation system can find the optimal plan for the overall function of the planning system based on the evaluation of various parts, levels, and subsystems. During the overall optimization process, it continuously provides relevant information to decision-makers. There is a basis for urban public transportation systems and the screening of urban public transportation planning schemes.

\section{Correlation Analysis of Environmental and Social Problems in the Transportation System}

2.1. Relationship between Environmental and Social Issues. The carrying capacity of the urban transportation system is based on various traffic volumes, the average carrying capacity of different vehicles is different, and the traffic volume occupied by the unit traffic volume is also different. The resources required by individual motor vehicles for sharing are far more than other types of traffic. The situation of way sharing shows that the efficiency based on resource utilization is very low. Also, for different vehicles, due to the difference in average carrying capacity and technical indicators such as environmental pollution and energy consumption, it will lead to different natural resource consumption per unit of transportation. In the case of a certain total demand for transportation trips and the difficulty of road reconstruction and expansion, the share of high-capacity transportation modes in the share of residents' trips has a more lasting and effective effect in managing traffic problems than the method of expanding transportation supply [18]. The effect of controlling traffic problems is more durable and effective than blindly expanding the supply of traffic, and it is also more effective in promoting the harmonious development of man and nature, as shown in Figure 1.

To achieve the sustainable development goals of the historical block transportation and the historical block transportation structure that meets the requirements of social, the historical and cultural characteristics of the block and its sustainability are combined. The purpose of this paper is to establish the relationship between the microindividual travel mode selection behavior and the travel characteristics of residents in historical blocks, the traffic policies in the blocks, and the characteristics of different traffic modes in the blocks. From the macro- and microlevel study, the evolution mechanism of the traffic structure established the proper traffic structure of the blocks based on the sustainable development of social, economic, and environmental systems. To put forward the evaluation system and methods for the optimization of the traffic structure of historical blocks, the green important reference is sustainable for development.

The urban public transportation system is a transportation system composed of various urban public passenger transportation modes. It mainly includes two aspects, that is, public transportation means and facilities and public transportation planning and operation. Public transportation means mainly include conventional buses, rapid rail transit, suburban railways, and public transportation stations. The urban public transportation system has unique characteristics that other urban passenger transportation vehicles do not have.

The capacity of both public transit and rail transit in urban public transportation is dozens or even hundreds of times that of ordinary transportation. The wide range of its services provides a large number of customer groups for the realization of large-volume services. Therefore, whether 


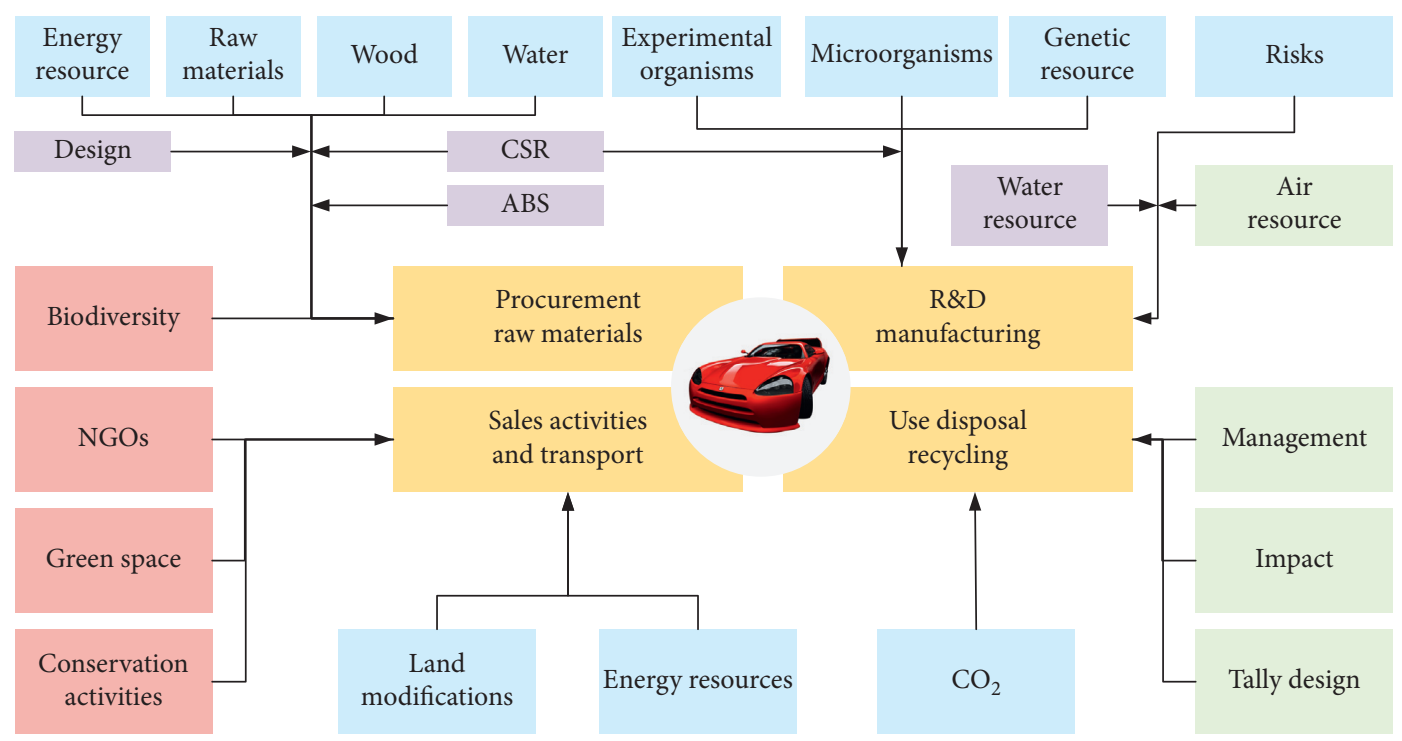

FIGURE 1: Relationship between environmental and social issues.

from the perspective of public transportation itself or the perspective of its service groups, a large volume is the basic feature of the development of the urban public transportation system.

This feature is complementary to the feature of a large volume. In the case of a certain number of public transportation vehicles, their large capacity and wide coverage of operating lines can effectively meet customer needs under the condition of fewer operating time, improve road utilization, and save road space. The technical level of drivers in the urban public transportation system is strictly controlled by the transportation department of the urban public transportation system, which effectively guarantees the safe operation of vehicles. Besides, the driving range of public passenger transportation is within the range planned by the traffic control department, which ensures the road safety of public passenger transportation [19]. Each bus has to stop at bus stops that are scheduled on multiple lines, and there are often queues waiting to get on the bus. The constant stop and idle speed extend the idle time of the bus during operation. This is in turn produces more pollutant emissions. Buses need to stop at specific bus stops and traffic signals at many intersections continuously, and they need to continuously slow down, start, and accelerate. Increasing the starting frequency of the bus implementation process, the pollutant emissions generated during this process will also increase.

2.2. Transportation System Model Designs. The essence of the transportation system is a three-dimensional space curve with geometric invariance. Free movable frames are usually used to analyze the geometric characteristics of the threedimensional space curve in spatial differential geometry. For any three-dimensional space curve $r(s)$, at any arc length $s$, a unit tangent vector $T$, unit principal normal vector $N$, and unit subnormal vector $B$ can be determined. The formed
Frenet movable frame moves and rotates with the arc length $s$. The three coordinate planes determined by the Frenet frame are the close plane, the normal plane, and the second plane, and the relationship between the unit vectors at the arc length $s$ is calculated as follows:

$$
\left\{\begin{array}{l}
M(s)=\dot{r}(s)=\frac{\mathrm{d} r}{\mathrm{~d} s}, \\
N(s)=\dot{T}(s), \\
B(s)=M(s)^{*} N(s) .
\end{array}\right.
$$

The Frenet frame changes with the arc length $s$. The Frenet-Serret formula expresses this change:

$$
\left\{\begin{array}{l}
M(s)=\dot{\kappa}(s) \dot{N}(s), \\
Y(s)=\dot{T}(s) \dot{\kappa}(s) N(s), \\
B(s)=-M(s)^{*} N(s) .
\end{array}\right.
$$

Therefore, there is a formula for calculating the curvature and deflection of the space curve at the arc length $s$ :

$$
\dot{\kappa}(s)=\left|\frac{\mathrm{d} T}{\mathrm{~d} s}\right|=\frac{|r(s) \times r(m)|}{|r(n)|^{6}} .
$$

Curvature and deflection are used as the basic description indicators of the space curve. The former represents the rotation speed of the tangent vector of the curve to the arc length, that is, the degree of curvature of the curve. The latter describes the rotation speed of the second normal vector of the curve to the arc length, namely, the curve degree of torsion [20]. When the deflection rate is 0 , it represents a plane curve. The change of the Frenet frame at any point on the curve can be uniquely determined by the curvature and torsion of the curve; that is, when the 
expressions of the curvature and torsion about the arc length and the starting point of the curve are given, the change of the Frenet frame is determined on the curve. The geometry of the curve is determined accordingly.

The road environment is one of the important factors affecting the driving behavior of the driver. The continuous monotonous landscape will induce the driver to fatigue driving, and the complex and changeable landscape will increase the driving load of the driver. However, environmental factors are rarely extracted by parameters at present, so that there is little quantitative analysis of the relationship between environmental factors and accidents. To this end, this study uses computer vision technology to perform image processing on the driving video and obtains the characteristic indicators of the environmental factors during the actual driving of the driver from the image, including the sky scale index, road surface scale index, roadside scale index, and brightness index. This section introduces in detail the extraction process of each environmental factor characteristic index, as shown in Figure 2.

Comparison between different bus planning schemes is in the same city. If the city has not yet established a public transport network, it is still in the planning stage, and a decision-making unit is several planning schemes. If the city itself has already established a public transportation network, the decision-making unit is the current basic scheme and other planning schemes. Comparison of public transportation systems between different cities are in the same city in different years. This situation is mainly to evaluate the adaptability of public transportation to urban development and road network level. The decision-making unit is the integrated transportation system of different cities or the integrated transportation system of the same city in different years.

The second step is to determine the DEA input and output system, which mainly depends on the evaluation dimension selected by the decision-maker. It is assumed that the decision-maker has selected $m$ input indicators and $s$ indicators, and the comparison between these indicators is obtained. Judgment matrixes $\mathrm{C}$ and $\mathrm{B}$ are provided with $n$ decision-making units. Input and output values are known to the index value, $X$ represents the input index value. $Y n$ represents the output indicator value. The weight of the input and output indicators is used as a variable, and then the efficiency index of the jet decision unit is

$$
\beta_{j}=\frac{U^{T} Y_{j}}{V^{T} X_{j}} .
$$

The AHP preference cone is added to the constraints and the CCR model is constructed as follows:

$$
\begin{aligned}
H= & \operatorname{Mex}_{0} \sum_{i=0}^{\infty} \alpha^{i}\left(\frac{H_{i}^{1-\delta}}{1-\delta}-\frac{L_{t}^{1+\varphi}}{1+\varphi}\right), \\
K_{t}= & {\left[\left(1-\phi_{1}\right)^{1 / \phi}\left(K_{F, t}\right)^{(p-1) / p}\right]^{(p-1) / p}, } \\
\operatorname{MHP}_{\mathrm{Bal}_{i}}^{d}= & {\left[\frac{\left|\mathrm{TTM}_{i}^{q}-\mathrm{TCM}_{i}^{n}\right|}{\sqrt{\mathrm{TCM}_{i}^{q}+\mathrm{TCM}_{i}^{n}}}\right]^{-4}, } \\
& -\kappa_{t}^{\varphi}+\eta_{t} W_{i, t}=0, \\
G_{t}^{*}= & {\left[\left(1-\phi_{1}\right)^{1 / \phi}\left(M_{F, t}\right)^{(p-1) / p}+(1-\gamma)^{1 / \phi}\left(M_{F, t}\right)^{(p-1) / p}\right]^{2} . }
\end{aligned}
$$

$G_{\mathrm{t}}$ is the corresponding energy value, $p$ is the value of the parameter $P$, and $t$ represents time. This formula means that the weight value of each decision unit is determined so that the decision unit has the maximum efficiency index, and the efficiency index of all decision units is not greater than one. After a series of line changes, equivalent linear planning problems are obtained and solved by programming. Many programming languages come with libraries to solve linear planning problems. As a function of the input parameters, the solution of a linear programming problem can be obtained.

The model in this paper changes according to user needs, that is, the selection of the index system, and the judgment matrix belongs to the input variables. The user needs to set the importance of the indexes in the selected input and output index system. The importance levels and assignments are shown in the table. There are $m$ input indicators and $s$ indicators in the evaluation scenario selected by the user which is shown in Table 1.

With the judgment matrix based on user input, a consistency check is also required to confirm that there is no much contradiction before and after the user's important input, to check the coordination between the importance of the indicators.

2.3. Evaluation of Examination Index. In the tranStar platform, the organizational form of multiprojects is adopted. As shown in Figure 3, this approach allows users to quickly generate solutions based on existing projects. In actual engineering projects, designers often have multiple sets of planning schemes and hope to get not only the implementation effects of several schemes but also the comparison of the effects of different schemes, and several schemes are based on the same road network database. Considering such a scenario, the software sets up a project file organization method, and there can be multiple program folders under a project, which provides convenience for the generation of 


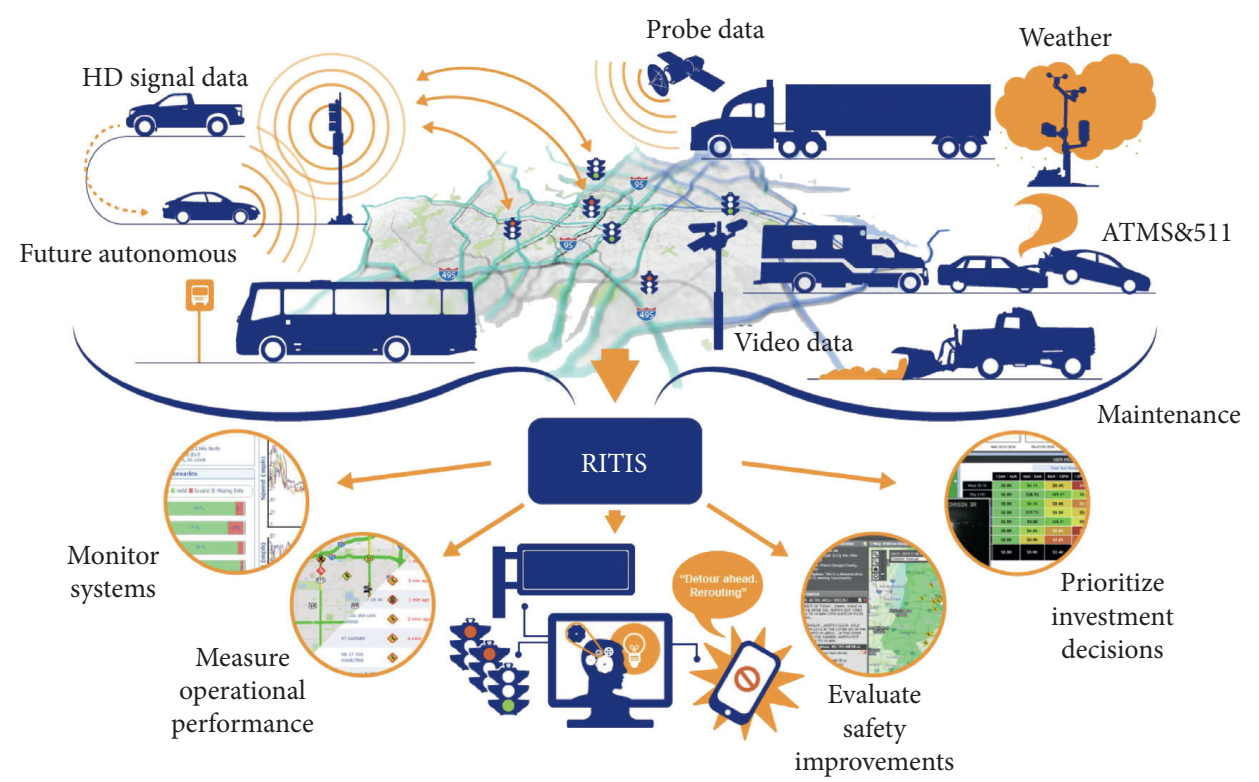

Figure 2: Schematic diagram of the traffic system model.

TABLE 1: Level 9 scale and meaning.

\begin{tabular}{lcc}
\hline Assignment & Values & Importance level \\
\hline 1 & 5.6 & $i$ and $j$ are equally important \\
3 & 4.7 & $i$ and $j$ indicators are slightly more important \\
5 & 6.4 & $i$ and $j$ indicators are important \\
7 & 2.5 & $i$ and $j$ indicators are strongly important \\
9 & 4.7 & $i$ and $j$ indicators are extremely important \\
$2,4,6,8$ & 6.8 & The scale value corresponding to the intermediate state \\
\hline
\end{tabular}

new programs and the comparison between programs. This paper is to establish an evaluation and decision module based on the organizational form of such a multiproject project system. In the program design, it is necessary to quickly read the basic road network data, public transportation network data, and passenger flow distribution data files in tranStar format, which will be read into the data which are converted into computable variables.

Each instrument has an event recording function, such as the intelligent vehicle-mounted intelligent detector that can record events such as vehicle ignition, speed, and throttle pressure. The biopsychological detector can define event markers through the data collection platform to record vehicle ignition, vehicle start, vehicle passing through the whole pile section, the vehicle passing through the tunnel opening, and other events. Eye trackers and driving recorders have video recording and sound recording functions and can also record events such as vehicle ignition, vehicle passing through the whole pile section, and vehicle passing through the tunnel opening section. Therefore, the same event recording time is benchmarked by the event recording function of each instrument to complete the time alignment of various data. Since the instrument with the lowest sampling frequency among all the instruments is a smart box with excellent driving, the sampling frequency is $2 \mathrm{~Hz}$. Therefore, after

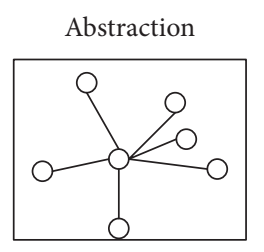

Orientation

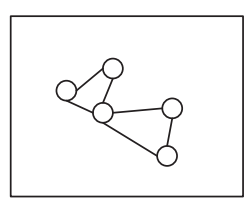

Pattern

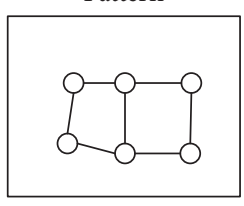

Figure 3: Evaluation steps of verification index.

time-aligning the data collected by each instrument, the time series data are output in $0.5 \mathrm{~s}$ time steps.

The input of the evaluation decision module has two parts. One is the user's input on the parameter setting interface. The software will read the user's input and store it in 
the DEA model parameter table. The DEA model parameter table is mainly about which input-output indicators and weight judgment matrix are selected. The built-in software will number each indicator, so the parameter table is all numbers, the first line is the input index number, the second line is the output index number, the third line is the input weight index judgment matrix, and the fourth line is the output index weight judgment matrix. The second part of the input depends on the virtual transportation system's oneclick simulation results of multiple schemes. It will input the traffic volume predicted by the road section for each transportation mode, the passenger flow distribution of the bus line network, and the amount of red on the bus station. These are the output of other modules of the platform and the input of the evaluation decision module. We read these related files to calculate the evaluation indicators and output them to the overall urban transport evaluation function report on each program.

The input and output indicators of the decisionmaking in the index report of each program are selected, use the parameter table of the DEA model and the inputoutput indicator values of each program as the input of the DEA model and then calculate, and the software then outputs the efficiency index of each solution and the solution ranking results to the index report file for users' reference [21]. The future development direction of urban space can guide the expansion of the city, and urban transportation is an important support for the development of urban space. Large railway passenger station areas with multiple transportation modes are more accessible than other areas of the city. Future development prospects are also greater. At the same time, urban development generally concentrates better resources on the future development direction of the city. Therefore, the location of railway passenger stations should be controlled in coordination with urban space and transportation, and priority should be given to the future development direction of urban space or the centralized distribution of transportation points to promote the integrated development of passenger stations and cities.

\section{Results and Analysis}

3.1. Environmental and Social Issues Affect Results. To verify the operation effect of the SD model, that is, whether the operation of the model can be maintained at different time steps, the operation stability of the model can be maintained. The operation step size of the model is specially set, and the setting steps are 0.25 years, 0.5 years, and 1 year, as shown in Figure 4.

In the case of step sizes of $0.25,0.5$, and 1 year, the model runs have a certain difference except for the number of runs, and the numerical results almost overlap. Under these three scenarios, the public transport pollutant measurement results of this model are roughly the same as the actual pollutant measurement trend. Therefore, the model meets practical requirements. Therefore, in general, the changing trend of the simulation results under the medium step size

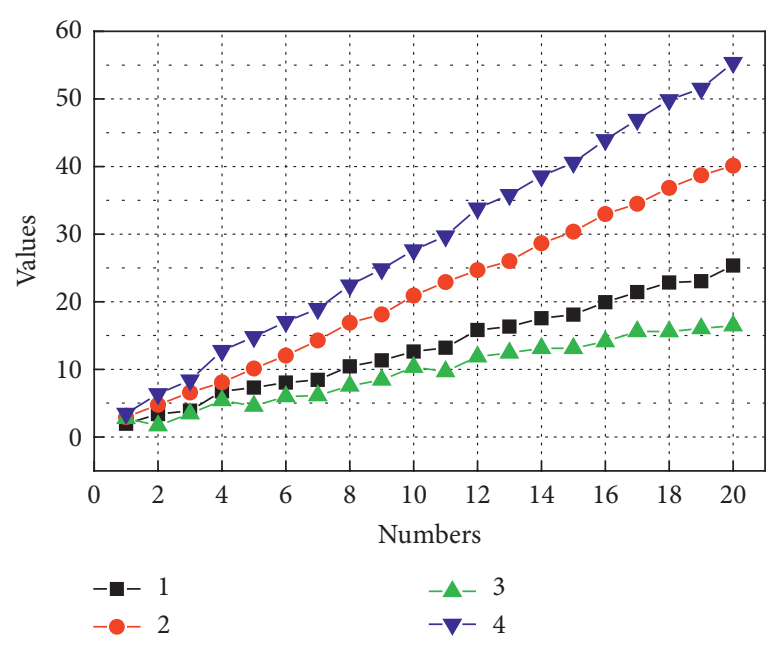

FIgURE 4: Invisible results of environmental and social issues.

tends to be consistent, which meets the requirements of the model for stability.

When applying the regression model to the actual project, the researchers are most concerned about whether the regression model predicts the reliability of the accidentprone sections. It is the core task of highway accident analysis and prediction to control and eliminate frequent accident sections. Only when the accident prediction model can be effectively used to predict frequent accident sections, it can play a good role in improving the safety level of the highway. To this end, we obtain the accident-prone sections in the test sections through traditional accident statistical analysis methods. Then, by comparing the matching degree between the prediction results of the regression model and the actual accident-prone sections, the engineering practicability of the regression model is investigated. As shown in Figure 5, according to the cumulative frequency curve method, it can be concluded that the road segment unit with more than accidents in the test road segment should be regarded as a road segment with frequent accidents.

The time distribution characteristics of the travel chain can reflect the peak travel period in the historical block, the general lifestyle of travelers, and travel laws and can better help determine the peak travel time of the day. The time distribution characteristics of the travel chain are affected by the travel chain mode, and the generation of the travel chain mode is directly related to the traveler's daily activities. The occurrence time of every single trip in the travel chain is related to the purpose of the trip, and the traveler performs a single trip in the travel chain separately according to his daily activity schedule [22]. Taking computers as an example, the daily commuting time of commuters is relatively fixed. The daily lunch break of urban residents is also relatively fixed, so the travel needs of various periods have certain trends and laws due to the residents' travel purpose and living habits.

3.2. Performance Analyses of the Traffic Model. As shown in Figure 6, for all trips based on home, the whole presents a strong peak, the peak duration is long, the peak is large, and 


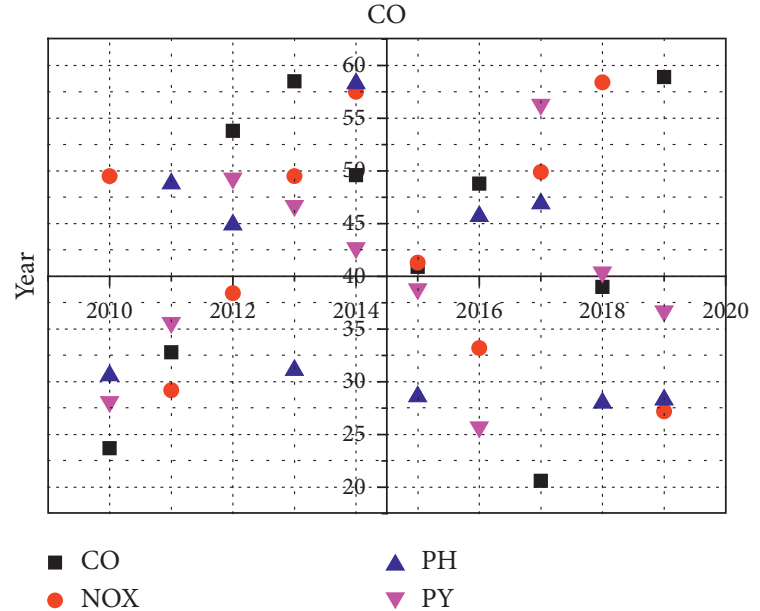

FIgURE 5: Results under different factors.

its travel demand reaches the highest value at $7: 30$, which is similar to the peak travel demand time of the sample overall. In agreement, for all home-based travel, the high travel demand lasts from $6: 30 \mathrm{am}$ to $10: 30 \mathrm{am}$, indicating that in addition to the majority of commuting needs. The residents of the historic district also have a considerable portion of shopping or leisure travel needs. For workplace-based travel, it has a peak travel time showing large and small 2 trend peaks, which shows a small peak at noon, as travelers have to go home at noon. The demand for lunch breaks is the commuting time of most commuters, which coincides with the overall travel evening peak of the sample.

The change process of road and environment during driving is the dynamic change process of the stimulus information feature index set. In other words, any feature index in the perception reaction layer is affected by the stimulus information layer as a whole. When establishing the VAR model of the stimulus information layer and the perception response layer, the stimulus information layer is regarded as a whole, and the impact of changes in this whole on the factors in the perception response layer is discussed [23]. Therefore, it is necessary to determine the optimal lag order of the VAR model between the whole stimulus information layer and the different sensory response layer feature indicators. It is selected by the largest number of criteria lag orders as the optimal lag order, with the establishment of the corresponding VAR model. As shown in Figure 7, when the lag order is 10, the VAR model obtains the lag order of the stimulus information layer of the normal road section and the perceptual response layer of individual feature indicators, such as LL, LR, FPE, AIC, and SC.

VAR model lag operator polynomial roots should be less than 1 . The AR feature root test was used to test the stability of the stimulus information layer-perceptual response layer of VAR model in normal road sections. Each VAR model selected the corresponding order. The AR root graph is introduced to visually illustrate the stability results of each VAR model. As shown in Figure 8, the VAR model is stable for a single feature index of the stimulus information layerperception response layer.

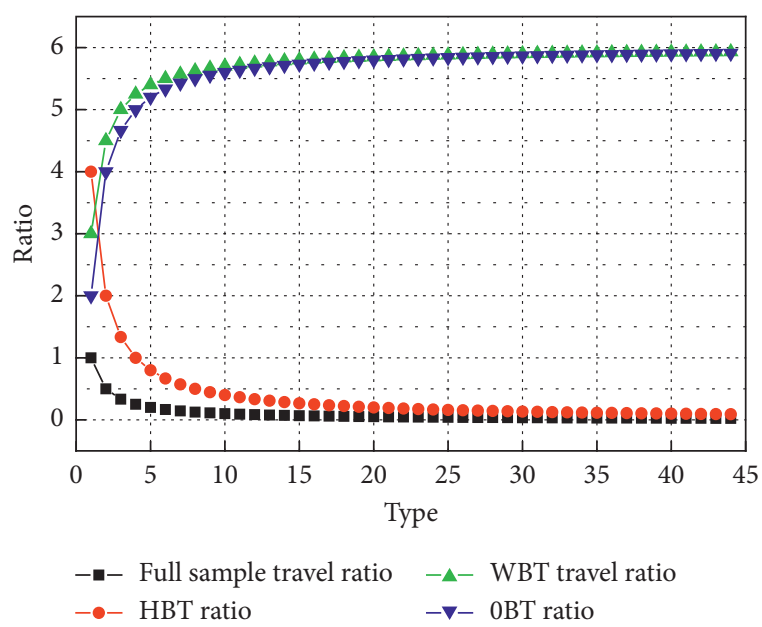

Figure 6: Statistics of travel time distribution of typical activity modes.

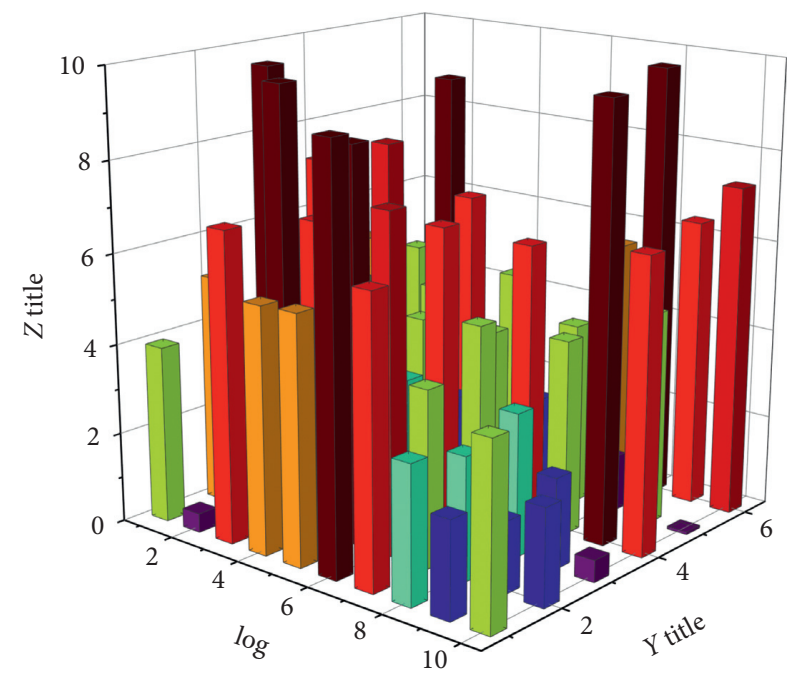

Figure 7: Statistics of lagging order of normal traffic system stimulus information layer and environmental and social problems.

When the independent variable set in the VAR model passes the Granger test for the dependent variable, it can be explained that the stimulus information layer is the Granger reason for the corresponding characteristic index of the perception response layer, and it also shows that the lagging change of the stimulus information layer has lagging changes which have predictive power. Currently, the VAR model can be used to analyze the factors in the stimulus information layer using impulse response functions and variance decomposition methods, and it is meaningful to analyze how the results affect the characteristic indicators in the perceptual response layer.

3.3. Analysis of Coordination Results. The coordinated response function analysis is mainly used to quantitatively analyze the impact of the change of the upper layer's characteristic index on the next layer's characteristic index. 


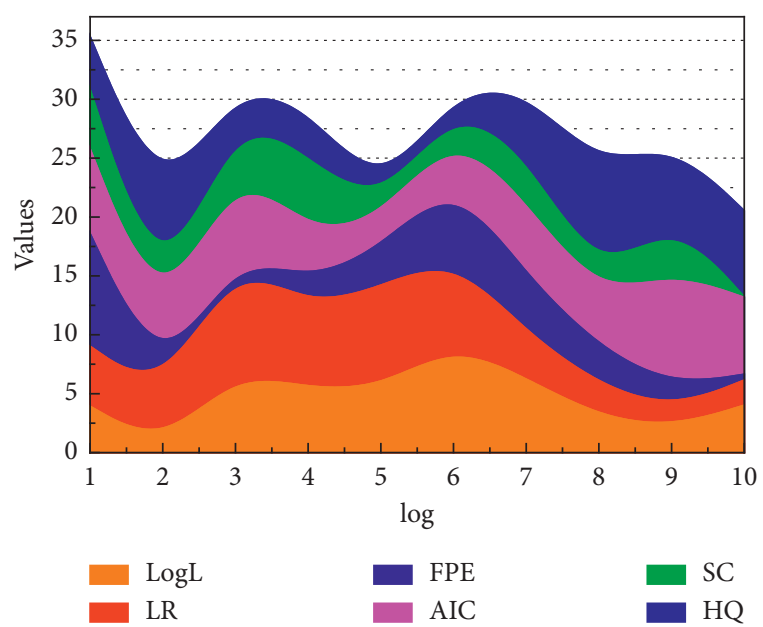

Figure 8: Normal traffic system stimulus information layer: perception response layer.

The method is to apply a unit standard deviation pulse to a certain characteristic index in the upper layer to investigate the next layer and the response of the characteristic index of this change. Therefore, impulse response function analysis is performed on each characteristic index of the normal road stimulus information layer and the perception response layer that passed the Granger test. As shown in Figure 9, it is the heart rate, skin conductance response, fixation ratio, and fixation time in the perception response layer.

It shows the link to the normal curvature of a positive pulse standard deviation. When the curvature increases, the driver's heart rate will increase because the curvature increases rapidly, the degree of curvature of the road section where the driver is located increases, and the increased pressure on the driver will naturally increase the driver's heart rate. However, overall, the driver's selfpsychological adjustment will restore the heart rate to a stable level. The adjustment period is 12 periods. In other words, the driver can adjust the self-adjustment to dissipate the curvature of space caused by the spatial curvature within the $500 \mathrm{~m}$ travel range. A standard deviation reduced the maximum amplitude; then the amplitude decreases rapidly in the first 3 cycles which converges to 0 . When the brightness increases, the driver's heart rate will decrease because the increase in brightness will improve the driver's observation of the environment, reduce the pressure on the driver caused by the environment, and naturally reduce the driver's heart rate. However, the pulse effect of brightness on the driver's heart rate dissipates within $150 \mathrm{~m}$ of travel.

As shown in Figure 10, the differences in the exposure levels of the four pollutants of the three populations are relatively stable. Taking $\mathrm{PM}_{10}$ as an example, the analysis found that the difference in the exposure level of $\mathrm{PM}_{10}$ was 3.07 in 2016, but the performance value for it is 2.972 with a little change. Among them, the difference between $\mathrm{CO}$ and NOX is large which are both in 24.25-37.37, and the difference between $\mathrm{PM}_{2.5}$ and $\mathrm{PM}_{10}$ is small.

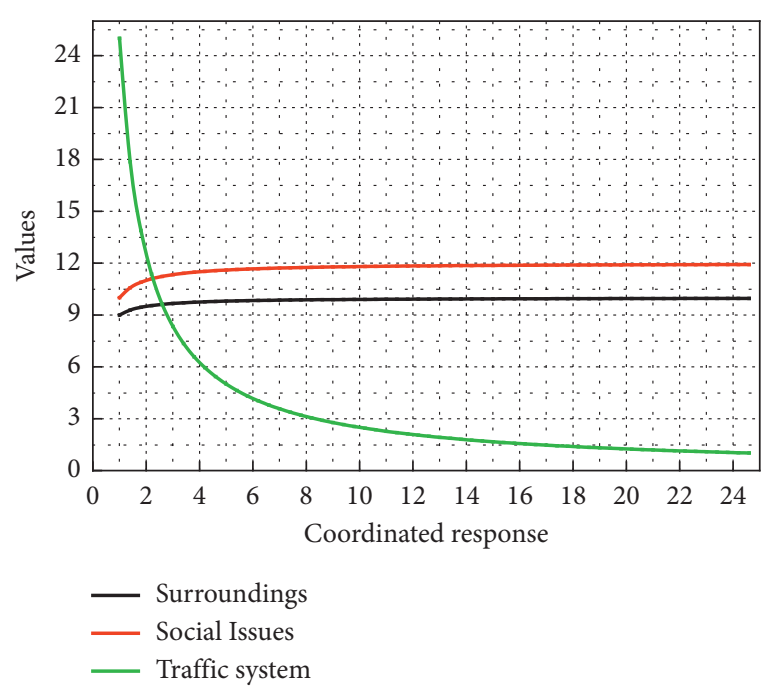

Figure 9: Coordinated response diagram of environmental and social issues to the information layer of the transportation system.

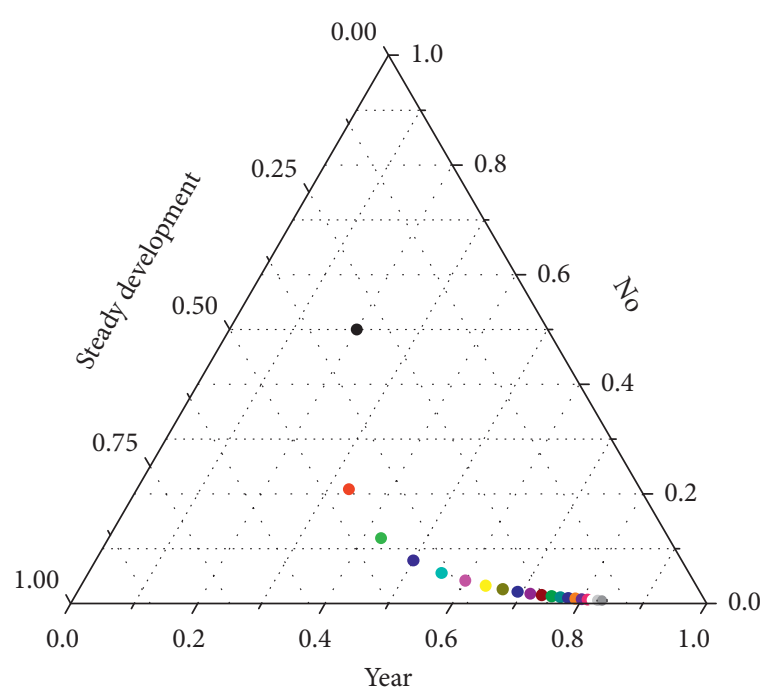

FIGURE 10: The results of environmental and social issues affecting the coordination of transportation systems.

In contrast, NOX has the largest exposure level difference in low-speed development and the smallest performance for high-speed development. Exposure levels of PM2.5 and PM10 to different populations are the same under the three scenarios. Besides, from this figure, we can see that the changes in the exposure levels of the four pollutants to different groups of people gradually became smaller, indicating that the differences in the exposure levels of the four pollutants for different groups of people gradually decreased [24]. The reason is that, on the one hand, as the exposure level continues to decrease, the difference in exposure levels of different populations decreases accordingly. The travel frequency of primary and middle school students and the elderly has also increased, making the health of different types of resident wind risk exposure differences continue to decrease. 


\section{Conclusion}

This paper first analyzes the characteristics of the transportation system and builds a multidimensional transportation evaluation index system from the perspective of basic line network evaluation, adaptability evaluation, and different users. The applicability of the data envelopment analysis model of common models for efficiency evaluation was studied, and the coordination system of the DEA efficiency evaluation model was constructed based on environmental and social issues. The BCC model was selected to evaluate the level of public transport construction and urban development and road network. For the case where the decision unit is not enough, the DEA model can be added with preference to come to restrict the weight. The DEA used in this paper has different input and output indicators in different decision-making scenarios, but it also needs to consider the preference of the decision-makers themselves. The AHP preference cone can solve this problem and add the decision-makers to the original DEA model. Own preference factors and the evaluation results can solve the problem of an insufficient number of decision-making units. At the same time, the AHP evaluation method has a strong subjectivity, and the evaluation results are completely determined based on artificial preferences. The traditional DEA model is too dependent on the data and requires many effective sample sets. It cannot reflect the preference of the decision-maker. The DEA method can be made more practical.

\section{Data Availability}

The data used to support the findings of this study are available from the corresponding author upon request.

\section{Conflicts of Interest}

The authors declare no conflicts of interest.

\section{Acknowledgments}

This work was supported by the Chinese Philosophy and Social Sciences Planning Office: Study on Coping Mechanism of Ecological Desertification of Traditional Village Culture in Urban-rural Integration (no. 18BSH089).

\section{References}

[1] M. G. Guzman, M. Alvarez, and S. B. Halstead, "Secondary infection as a risk factor for dengue hemorrhagic fever/dengue shock syndrome: an historical perspective and role of antibody-dependent enhancement of infection," Archives of Virology, vol. 158, no. 7, pp. 1445-1459, 2013.

[2] Y. Yin, "Multiobjective bilevel optimization for transportation planning and management problems," Journal of Advanced Transportation, vol. 36, no. 1, pp. 93-105, 2002.

[3] F.-Y. Wang, "Parallel control and management for intelligent transportation systems: concepts, architectures, and applications," IEEE Transactions on Intelligent Transportation Systems, vol. 11, no. 3, pp. 630-638, 2010.
[4] G. Currie, "Quantifying spatial gaps in public transport supply based on social needs," Journal of Transport Geography, vol. 18, no. 1, pp. 31-41, 2010.

[5] L. Redman, M. Friman, T. Gärling, and T. Hartig, "Quality attributes of public transport that attract car users: a research review," Transport Policy, vol. 25, no. 1, pp. 119-127, 2013.

[6] V. Van Acker, B. Van Wee, and F. Witlox, "When transport geography meets social psychology: toward a conceptual model of travel behaviour," Transport Reviews, vol. 30, no. 2, pp. 219-240, 2010

[7] D. Zeng, H. Chen, R. Lusch, and S.-H. Li, "Social media analytics and intelligence," IEEE Intelligent Systems, vol. 25, no. 6, pp. 13-16, 2010.

[8] R. Borndörfer, M. Karbstein, and M. E. Pfetsch, "Models for fare planning in public transport," Discrete Applied Mathematics, vol. 160, no. 18, pp. 2591-2605, 2012.

[9] Y. Liu, X. Liu, S. Gao et al., "Social sensing: a new approach to understanding our socioeconomic environments," Annals of the Association of American Geographers, vol. 105, no. 3, pp. 512-530, 2015.

[10] J. Ingram, "A food systems approach to researching food security and its interactions with global environmental change," Food Security, vol. 3, no. 4, pp. 417-431, 2011.

[11] Y. Quan and M. Sun, "Noticeable problems of transportation development in large metropolitan areas in China," Urban Transport of China, vol. 2, pp. 1-6, 2011.

[12] H. Haghshenas and M. Vaziri, "Urban sustainable transportation indicators for global comparison," Ecological Indicators, vol. 15, no. 1, pp. 115-121, 2012.

[13] J. S. Evans-Cowley and G. Griffin, "Microparticipation with social media for community engagement in transportation planning," Transportation Research Record: Journal of the Transportation Research Board, vol. 2307, no. 1, pp. 90-98, 2012.

[14] D. P. Willis, K. Manaugh, and A. El-Geneidy, "Cycling under influence: summarizing the influence of perceptions, attitudes, habits, and social environments on cycling for transportation," International Journal of Sustainable Transportation, vol. 9, no. 8, pp. 565-579, 2015.

[15] S. Dorado and M. J. Ventresca, "Crescive entrepreneurship in complex social problems: institutional conditions for entrepreneurial engagement," Journal of Business Venturing, vol. 28, no. 1, pp. 69-82, 2013.

[16] S. Kim, "Assessing mobility in an aging society: personal and built environment factors associated with older people's subjective transportation deficiency in the US," Transportation Research Part F: Traffic Psychology and Behaviour, vol. 14, no. 5, pp. 422-429, 2011.

[17] J. Yang, "Spatial and social characteristics of urban transportation in Beijing," Transportation Research Record: Journal of the Transportation Research Board, vol. 2193, no. 1, pp. 59-67, 2010.

[18] M. H. Mansourianfar and H. Haghshenas, "Micro-scale sustainability assessment of infrastructure projects on urban transportation systems: case study of Azadi district, Isfahan, Iran," Cities, vol. 72, pp. 149-159, 2018.

[19] C. Kormos, R. Gifford, and E. Brown, "The influence of descriptive social norm information on sustainable transportation behavior," Environment and Behavior, vol. 47, no. 5, pp. 479-501, 2015.

[20] Y. Fan and C. Liu, "Solving stochastic transportation network protection problems using the progressive hedging-based method," Networks and Spatial Economics, vol. 10, no. 2, pp. 193-208, 2010. 
[21] A. Crudden, K. Antonelli, and J. O’Mally, “Transportation self-efficacy and social problem-solving of persons who are blind or visually impaired," Journal of Social Work in Disability \& Rehabilitation, vol. 15, no. 1, pp. 52-61, 2016.

[22] L. Seliske, W. Pickett, and I. Janssen, "Urban sprawl and its relationship with active transportation, physical activity and obesity in Canadian youth," Health Reports, vol. 23, no. 2, pp. 17-25, 2012.

[23] F. J. Martinez, C. K. Chai-Keong Toh, J.-C. Cano, C. T. Calafate, and P. Manzoni, "Emergency services in future intelligent transportation systems based on vehicular communication networks," IEEE Intelligent Transportation Systems Magazine, vol. 2, no. 2, pp. 6-20, 2010.

[24] J. Faulin, A. Juan, F. Lera, and S. Grasman, "Solving the capacitated vehicle routing problem with environmental criteria based on real estimations in road transportation: a case study," Procedia-Social and Behavioral Sciences, vol. 20, no. 4, pp. 323-334, 2011. 